\title{
Alternativas de comunicación para redes de sensores AMI en Internet de las cosas para escenario energético en ciudades inteligentes
}

\author{
Communication alternatives for AMI sensor networks \\ in the Internet of things for an energetic scenario in smart cities \\ Fernando Ulloa-Vásquez ${ }^{1 *} \quad$ Dante Carrizo ${ }^{2} \quad$ Luis García-Santander ${ }^{3}$ \\ Recibido 5 de agosto de 2020, aceptado 16 de octubre de 2020 \\ Received: August 5, 2020 Accepted: October 16, 2020
}

\begin{abstract}
RESUMEN
Las tecnologías de comunicación aplicadas en soluciones con Redes de Sensores Inalámbricos, Internet de las Cosas y Máquina a Máquina, son los pilares fundamentales para un escenario considerando gestión energética. Este trabajo, se centra en aplicaciones para el logro de Ciudades Inteligentes que esperan priorizar la calidad de vida a través de aplicaciones de Tecnologías de Información y Comunicaciones. Para ello, se definen los principales conceptos del tema en estudio para posteriormente realizar una identificación específica en el escenario energético con respecto a las tecnologías de comunicación incluyendo las alarmas de emergencia y datos enviados por Medidores Inteligentes. Finalmente se analizan y comparan las Tecnologías-Estándares más adecuados que se aplicarán a cada tipo de infraestructuras en el escenario energético.
\end{abstract}

Palabras clave: Ciudades inteligentes, tecnologías de comunicación, Internet de las cosas.

\begin{abstract}
The communication technologies applied in solutions with Wireless Sensor Networks, Internet of Things, and Machine to Machine are the fundamental pillars for a scenario considering energy management. This work focuses on applications for the achievement of Smart Cities that hope to prioritize the quality of life through applications of Information Technology and Communications. To do this, the main concepts of the subject under study are defined to subsequently make a specific identification in the energy scenario with respect to communication technologies including emergency alarms and data sent by Smart Meters. Finally, the most appropriate Technologies-Standards that will be applied to each type of infrastructure in the energy scenario are analyzed and compared.
\end{abstract}

Keywords: Smart cities, communication technologies, Internet of things.

1 Universidad Tecnológica Metropolitana. Departamento de Electricidad. Santiago, Chile.

E-mail: fulloa@utem.cl

2 Universidad de Atacama. Departamento de Ingeniería Informática y Ciencias de la Computación. Copiapó, Chile. E-mail: dante.carrizo@uda.cl

3 Universidad de Concepción. Departamento de Ingeniería Eléctrica. Concepción, Chile. E-mail: luis.garcia@udec.cl

* Autor de correspondencia: fulloa@utem.cl 


\section{INTRODUCCIÓN}

Los nuevos requerimientos para obtener una Infraestructura Avanzada de Medición (Advance Metering Infrastructure, AMI), es posible gracias a un nuevo paradigma de comunicación y a los avances en los protocolos de internet. Esto permite la comunicación entre máquinas, que son posibles de implementar de manera más sencilla que hace décadas, donde los dispositivos comúnmente empleados no eran compatibles entre sí y no presentaban tantas opciones tecnológicas de comunicación [1-4].

Para la implementación de las tecnologías a los distintos sectores de la red eléctrica inteligente, se necesita una integración de diferentes redes heterogéneas para lograr un sistema interconectado eficiente. En Figura 1, se ilustra una descripción general de componentes a lo largo de toda la red y la infraestructura integrada desde Generación, Transmisión y Distribución eléctrica. El objetivo es lograr obtener los siguientes resultados:

- AMI, basadas en la automatización y recopilación de datos desde los medidores inteligentes (Smart Meter SM) con comunicación requerida en tiempo real.

- Automatización de viviendas, red Home Area Network HAN y de edificios inteligentes.

- Energías renovables no convencionales, distribuidas en áreas con servicio eléctrico urbano y rural. (Neighbor Area Network, NAN -Wide Area Network, WAN).
- Servicios públicos, centros de control de, subestaciones, empresas-mercados, proveedores de servicios que forman una red interactiva de múltiples agentes.

Este trabajo, se centra en aplicaciones para el logro de Ciudades Inteligentes que esperan priorizar la calidad de vida a través de aplicaciones de Tecnologías de Información y Comunicaciones. Para ello, se definen los principales conceptos del tema en estudio para posteriormente realizar una identificación específica en el escenario energético con respecto a las tecnologías de comunicación incluyendo las alarmas de emergencia y datos enviados por Medidores Inteligentes. Finalmente se analizan y comparan las Tecnologías-Estándares más adecuados que se aplicarán a cada tipo de infraestructuras en el escenario energético.

\section{INFRAESTRUCTURA DE INFORMACIÓN Y COMUNICACIONES INTEGRADAS RED ELÉCTRICA INTELIGENTE}

\section{Medidores Inteligentes}

Los SM se encargan de registrar los consumos eléctricos en domicilios, edificios e Industrias y son comunicados a una red interconectada que opera a través de una AMI. Esta infraestructura a menudo combina diferentes tecnologías de comunicación alámbricas (Power Line Communication, PLC, Broadband over Power Line, BPL) e inalámbricas (Celular, WiMAX, ZigBee). Los SM contienen

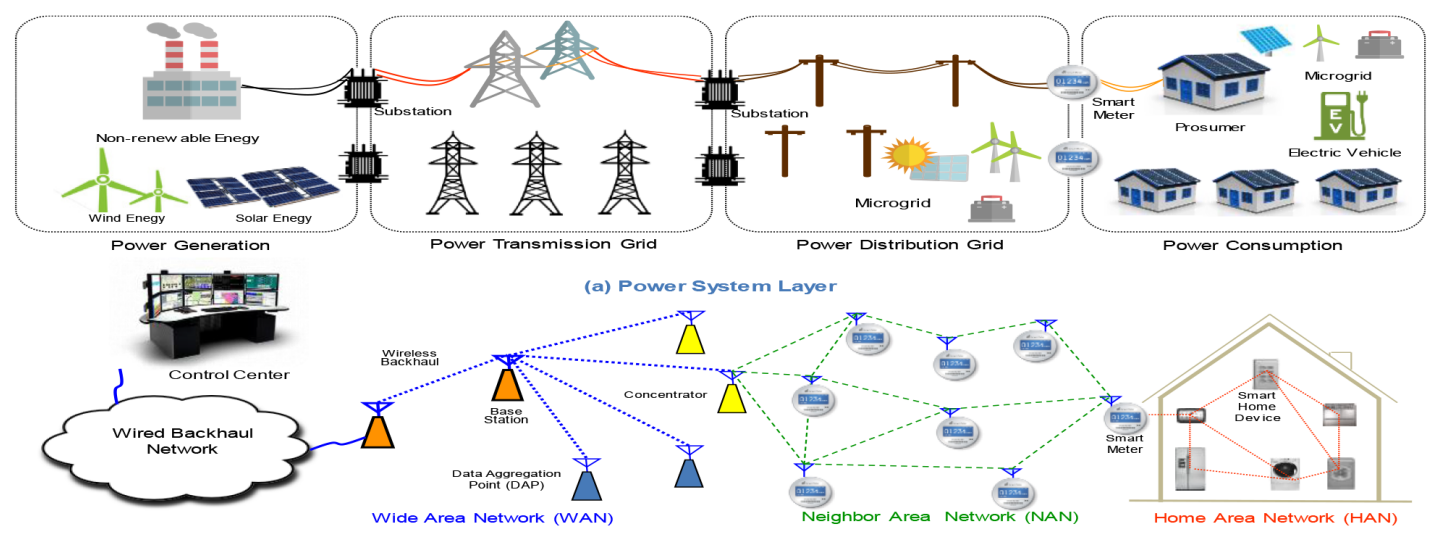

(b) Communications Layer

Figura 1. Infraestructura general de comunicaciones de la Red Eléctrica Inteligente. a) Energía Eléctrica. b) Capas de Comunicación [9]. 
interfaces integradas que admiten protocolos de conexión inalámbrica o alámbrica lo cual cumple con la infraestructura de la red. Dependiendo del tipo de red eléctrica, los hogares podrán contar con pantallas digitales o paneles táctiles, interfaz serial con un bus de comunicación para la gestión, además de incluir medidores de agua y gas. La optimización dinámica de precios-consumo y la gestión de remota del servicio, son un importante elemento que permite la adopción de esta nueva infraestructura AMI [5].

\section{Mediciones y confiabilidad en comunicaciones de Redes Eléctricas Inteligentes}

El principal indicador que se debe tener presente en redes de comunicación de computadores, máquinas, Smart Meter y Smart Socket (enchufes inteligentes), es la latencia. Esta se considera como el periodo de tiempo entre que ocurre una incidencia y el momento de corrección o acción del sistema en respuesta a tal incidencia. Los requisitos de latencia dependen de la aplicación en el contexto específico del sistema eléctrico de potencia. Los retrasos de comunicación de la red comprenden retrasos de transmisión, de propagación, de procesamiento (empaquetamiento, señalización y conmutación) y de puesta en cola como se muestra en Figura 2. Estos retardos aumentan la latencia de la red, por lo cual deben ser identificados comprendiendo el comportamiento completo de la red e identificando el retardo total de comunicación (Delay Error) [6].
La latencia de extremo a extremo que se puede alcanzar actualmente en Long Term Evolution, LTE con Internet por Operadora Celular, es cerca de 100 ms, (256 ms típico en redes convencionales de computadores) y no es suficiente para permitir aplicaciones de retraso crítico, relacionadas con el control o la protección de transmisión eléctrica (Ver Figura 3). Esta variación de frecuencia, como se muestra en Figura 3, es una medida tomada entre las dos estaciones [7] PMU (Phasor Measurement Units), UdA, UdeC y UTEM (proyecto Medfasee cono sur), en el evento ocurrido el 16 de Septiembre de 2015, con un sismo de 8.4 Grados Richter [8].

Este evento observado en Figura 3, tiene que tener una respuesta de los Teleoperadores y de las Teleprotecciones con valores inferiores a $10 \mathrm{~ms}$ como tiempos de respuesta. Comparado con los SM y Smart Socket que pueden tener una latencia del orden de varios segundos, ver Tabla 1.

Según la norma de distribución eléctrica IEC 61850, las operaciones de red automáticas rápidas requieren la entrega de mensajes de extremo a extremo en el orden de decenas de milisegundos o, incluso por debajo de $5 \mathrm{~ms}$. LTE permite comunicación M2M directa entre entidades de red inteligente cercanas entre sí, sin enrutamiento a través de la red central y cumpliendo este requisito de Latencia, al igual que lo cumple el estándar de comunicación WiMax $802.16 \mathrm{n}, \mathrm{h}, \mathrm{x}[9,10]$.

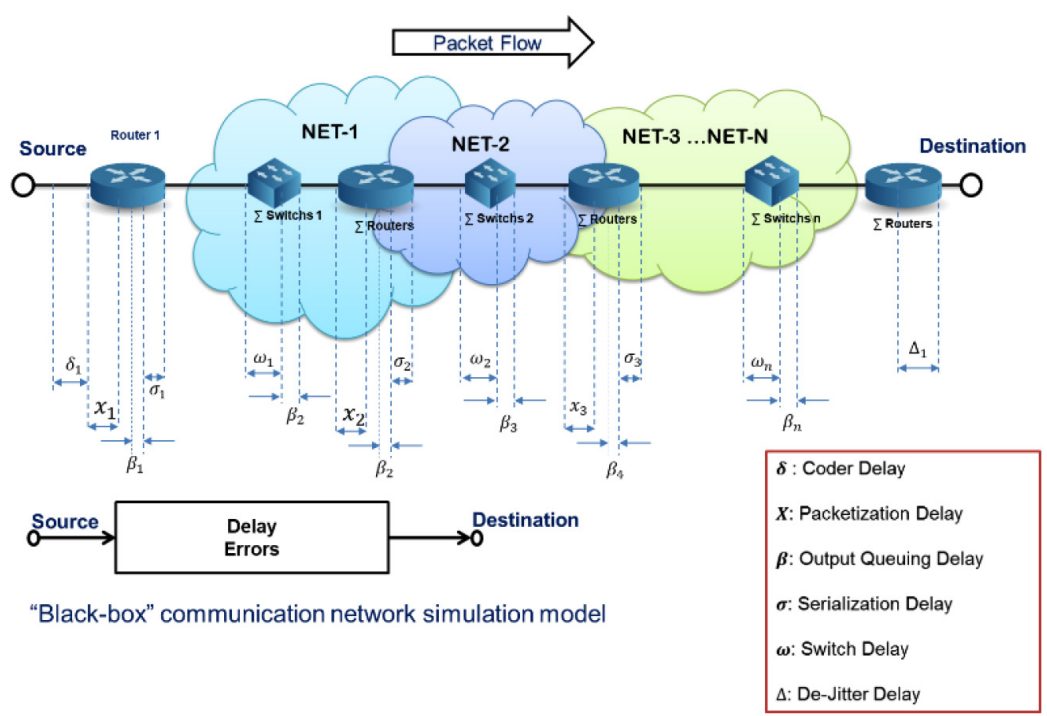

Figura 2. Tipos de retardos entre las redes de Internet [6]. 


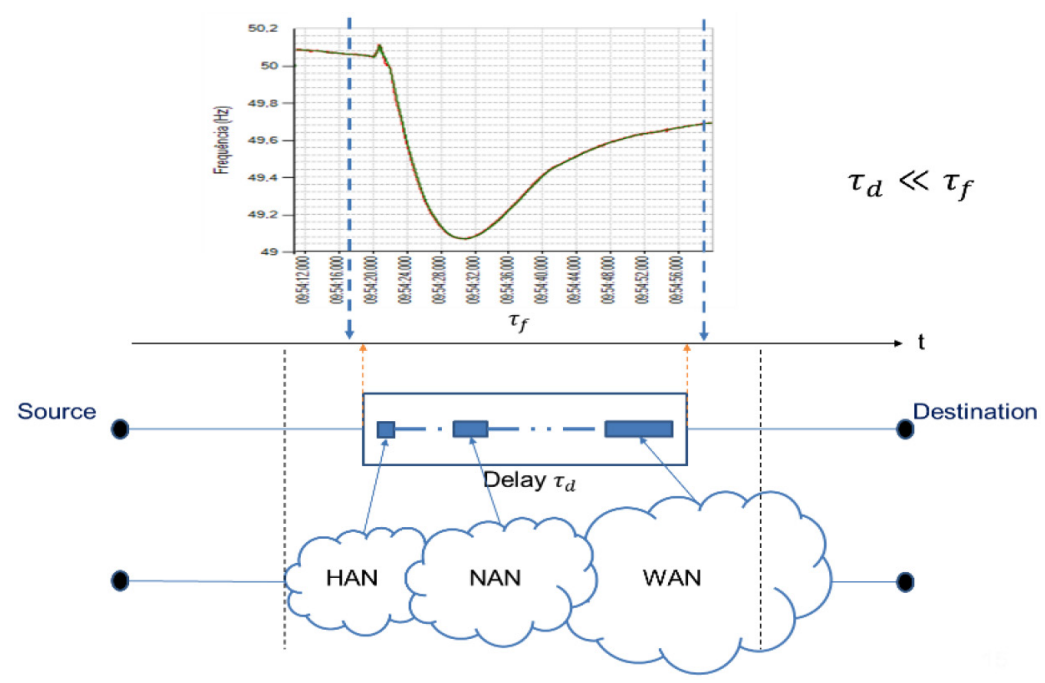

Figura 3. Comparación entre tiempo de falla $\mathrm{T}_{\mathrm{f}} \mathrm{y}$ tiempo de retardo $\mathrm{T}_{\mathrm{d}}$, en redes de comunicación de la Red Inteligente [8].

Tabla 1. Requisitos de latencia para redes eléctricas inteligentes.

\begin{tabular}{|l|c|}
\hline \multicolumn{1}{|c|}{ Application } & Maximum Latency \\
\hline Teleprotection & $\leq 10 \mathrm{~ms}$ \\
\hline Synchrophasor & $\sim 20 \mathrm{~ms}$ \\
\hline Control and data acquisition & $(100-200) \mathrm{ms}$ \\
\hline Smart metering & $(2-3) \mathrm{s}$ \\
\hline
\end{tabular}

\section{COMUNICACIONES EN LA RED ELÉCTRICA INTELIGENTE}

Para que estos sistemas de control y monitoreo logren aumentar la eficiencia y seguridad de la red, se requiere de una infraestructura de comunicación confiable y tolerante a fallos, combinando diferentes tecnologías para la transmisión, comunicación y almacenamiento de los datos, que permite al sistema ser sostenible en el tiempo. Este es el objetivo de la red inteligente. Comunicar a todos los sectores que colaboran en la red inteligente, requiere dividirla en redes más pequeñas para lograr una gestión conforme a las diferentes aplicaciones y contextos dentro de la red.

La red inteligente se divide en tres tipos de redes, cada una con una escala y un rango diferente:

- Las redes de área amplia (WAN) brindan comunicación entre la empresa suministradora de electricidad y las subestaciones, y como tales operan a media y alta tensión. Las WAN son redes de comunicación troncales con un ancho de banda de $100 \mathrm{Mbps}$, que permite manejar la transmisión de datos a larga distancia, por ejemplo decenas a cientos de $\mathrm{km}$.

- Las redes de área de campo (FAN) y las redes de área de vecindario (NAN) que permiten la comunicación entre algunos kilómetros y cientos de metros.

- Las redes de área doméstica (HAN), las redes de área de construcción (BAN) y las redes de área industrial (IAN) proporcionan comunicación entre los aparatos eléctricos y los medidores inteligentes dentro de la vivienda, el edificio o el complejo industrial.

La conexión de centrales, subestaciones y servicios públicos se ubican en el núcleo del sistema. En este sector son necesarias tecnologías de alta capacidad y disponibilidad de ancho de banda para operar con una extensa cantidad de datos que vienen de distintas partes del sistema y diferentes dispositivos terminales. Esta red troncal está compuesta generalmente sobre fibra óptica, ya que permite una alta velocidad de transferencia de datos y es inmune a interferencias.

La Infraestructura Avanzada de Medición (AMI) que permite la automatización de las subestaciones y sistemas de distribución en los centros de control, así como concentradores de datos de sistemas 
SCADA y SPMS [7], están presentes en la malla intermedia (Backhaul). Esta red, al igual que el núcleo, necesita proporcionar altos medios de ancho de banda ( $\leq 10 \mathrm{~ms}$, velocidad respuesta) y que las instalaciones de esta sean lo más sencillas y rentables posibles. Por la importancia de esta red es necesario que ésta tenga un flujo de datos flexible e ininterrumpido, ya que es importante que el transporte de datos sea confiable antes de ingresar al núcleo. Esta parte de la red inteligente contiene una mezcla entre tecnologías alámbricas e inalámbricas de comunicación y estas pueden ser WiMAX, 3G, 4G, LTE, fibra óptica, ondas milimétricas, BPL, entre otras.

El último extremo de la red inteligente está cobrando importancia, ya que en este sector se interactúa de manera directa con el usuario final. En este sector se consideran redes locales, tanto de hogares como de industrias, las cuales pueden estar o no conectadas con el servicio público. En este sector se encuentran aplicaciones de HAN (Home Area Network), BAN (Building Area Network) e IAN (Industries Area Network) que ocupan tecnologías compatibles para la automatización de hogares y edificios (domótica e inmótica). Éstas basan su comunicación principalmente en los estándares IEEE 802.15.4, IEEE 802.11x y PLC. Esta red procesa información relativa a la gestión de la energía en las edificaciones, que controla numerosos componentes como termostatos, aires acondicionados, dispositivos y sensores inteligentes, control de iluminación, vehículos eléctricos, generación distribuida, entre otros. La adquisición, recopilación, transmisión y análisis de los datos se realiza a través de aplicaciones IoT [11].

\section{Convivencia entre tecnologías y migración de IP}

La convergencia de diferentes protocolos de comunicaciones implica la correlación de datos de redes heterogéneas en toda la infraestructura. A continuación, se abordan varios problemas importantes antes de adquirir comunicaciones confiables:

1. Coexistencia: En redes, las variedades de tecnologías inalámbricas que comparten las mismas bandas de frecuencia, pueden incurrir en interferencias graves entre sí. El problema de la convivencia es crítico, ya que muchas redes inalámbricas en los hogares utilizan la misma banda, ISM de $2.4 \mathrm{GHz}$, de frecuencia para todas las comunicaciones al interior de la casa.
2. Interoperabilidad: Algunos dispositivos finales (SmartMeter) en redes heterogéneas pueden no estar asignados de forma única con direcciones IP globales. Por lo tanto, requerirán subcapas de convergencia implementadas en las puertas de enlace o enrutadores de borde para poder comunicarse entre sí. La puerta de enlace M2M convergente puede realizar la traducción de protocolos para diferentes tecnologías de comunicaciones, por ejemplo, PLC con IEEE 802.15.4. Se pueden encontrar ejemplos en la integración de Wi-Fi y WiMAX para el escenario de implementación en Last-Mile.

3. Integración de IPv6: Por otro lado, la interoperabilidad plantea un dilema sobre si asignar una dirección IPv6 global a cada dispositivo final o implementar una subcapa de convergencia para la conversión de protocolo/ dirección, por ejemplo, la traducción de direcciones de red (NAT) que se usa en las redes IPv4. Emplear cualquiera de ellos se basaría en las necesidades del mercado, así como en las estrategias y los requisitos de los proveedores de servicios y servicios públicos. Para el rendimiento general de la red inteligente, se requiere investigación adicional para lograr un alto rendimiento y una baja latencia.

\section{TECNOLOGÍAS SELECCIONADAS DE COMUNICACIÓN}

Los medios físicos utilizados para la transmisión de señales son el cobre, la fibra óptica y microondas. El medio físico más desplegado es el cobre debido a su antigüedad, le siguen las ondas de radio y microondas a causa del incremento de sus usos en las últimas décadas. Por último, están las tecnologías de comunicación por fibra óptica, las cuales se caracterizan por ser confiables, inmunes a interferencia electromagnética y proporciona grandes anchos de banda a distancias más largas, siendo perfecta candidata para el troncal de comunicaciones de un sistema tan grande y complejo como la Red Eléctrica Inteligente. La aplicación de éstas tecnologías dependerá tanto de la geografía como de los estándares de cada país o ciudad donde se quiera implementar la red, así también como las necesidades del sector empresarial para ofrecer los distintos servicios de esta nueva infraestructura y la necesidad de los usuarios finales [10]. 


\section{Tecnologías de comunicación óptica}

En esta clasificación se incluyen las tecnologías de fibra óptica y banda ancha sobre líneas eléctricas (BPL, Broadband Power Line). Estas tecnologías son muy utilizadas para las comunicaciones de servicios públicos, dependiendo de las zonas de disponibilidad de este servicio y cumplimiento de los requisitos de rendimiento. Las distintas tecnologías de fibra óptica son capaces de proporcionar grandes anchos de banda y velocidad de datos, estas pueden ser aplicadas en los cuatro subsectores mencionados en la sección anterior. Los avances en la red óptica síncrona de última generación y Jerarquía Digital Síncrona (Synchronous Digital Hierarchy, SDH), proporcionan soporte de suministro de varios servicios que admiten aplicaciones IP y Ethernet. A partir de lo anterior, se están integrando tecnologías de Conmutación de Etiquetas Multiprotocolo (Multiprotocol Label Switching, MPLS) en las redes de comunicación troncales existentes entre las subestaciones y los centros de control despacho de carga eléctrica. Esta tecnología logra el transporte de Ethernet a través de SDH en sus redes de conmutación existentes, mejorando la confiabilidad, la calidad de servicio y seguridad para las aplicaciones principales de la Red Inteligente. Las velocidades en este tipo de redes actualmente están llegando de los 40 a 100 Gigabit Ethernet $(\mathrm{GbE})$, con una gran capacidad de beneficios para el tráfico complejo del núcleo de la Red Inteligente [11].

\section{Tecnologías de comunicación alámbricas}

La infraestructura de comunicación de acceso al medio utilizado por PLC (Power Line Carrier Communication) tiene características homólogas con IEEE 802.3 Ethernet. Dentro de estas se pueden destacar las topologías en estrella orientadas a maestro-esclavo, token en red de anillo, bus con multiplexación por división de tiempo (TMD) y la técnica de acceso al medio con detección de colisiones (CSMA-CD). La principal ventaja de la comunicación por la línea de potencia se basa en que se puede aprovechar el cableado existente de las edificaciones, de manera que los dispositivos que necesiten suministro energético de la red también puedan comunicarse a través de esta, al estar conectados desde las tomas de corriente. La tecnología de BPL de Banda Estrecha (NB-PLC) generalmente se utiliza en las redes de media y baja tensión para comunicar Medidores inteligentes (Automatic Meter Reading o Smart Meters) instalados en los hogares o establecimientos, con los concentradores de datos ubicados en los transformadores desplegados en el sistema de distribución eléctrica, todo esto ubicado en la NAN (Neighborhood Area Network). Por otro lado, la tecnología BPL de Banda Ancha (BB-PLC) es utilizada para suministrar internet de banda ancha a través de un módem BPL que está conectado a cualquier punto en los hogares o edificios conectados a la red con PLC [12]. Dentro de las limitantes de estas tecnologías alámbricas tenemos:

- Impedancia variable y condición del canal cable cobre;

- No tiene, ruido blanco aditivo térmico;

- La atenuación dependiente de las ubicaciones geográficas. (Kilómetros);

- Cambio de fase (de monofásico a trifásico y viceversa) entre arquitecturas interiores y exteriores.

\section{Tecnologías Inalámbricas}

Las tecnologías de radiocomunicación digital han tenido un desarrollo creciente en las últimas décadas, lo que se refleja en la facilidad de implementación de éstas actualmente. Dentro de éstas tecnologías, están las redes celulares que principalmente se representan en tecnologías GSM, 3G, 4G, LTE, que están basadas en los estándares: Sistema Global para Comunicaciones móviles (GSM), CDMA2000 y el Sistema de Telecomunicaciones Móviles Universales (UMTS) [13, 14].

La tecnología de comunicación móvil celular GSM utiliza método acceso al medio de Acceso Múltiple por División de Tiempo (TDMA). Este tipo de tecnología mantiene su compatibilidad con las versiones anteriores al igual que General Packet Radio Service (GPRS), el cual suma sus capacidades de paquetes de datos y mejora las tasas de datos del sistema GSM para su evolución (EDGE), todo lo anterior estandarizado por la Third Generation Partnership Project (3GPP). La tecnología 3G, se basa en CDMA como lo es UMTS, también conocida como CDMA de banda ancha (WCDMA).

La tecnología de evolución a largo plazo (LTE) 3GPP pre-4G y el 4G-LTE Advanced, diseñado para aumentar la capacidad y la velocidad de sus redes celulares, han sido más atractivos para la adopción de operadores como AT\&T, T-Mobile y Verizon.

Una de las tecnologías capaces de ofrecer comunicación para la última milla es Wi-Fi, certificada por la 
Wi-Fi Alliance, basada en estándars $802.11 \mathrm{a} / \mathrm{b} / \mathrm{g} / \mathrm{h}$, los cuales operan en las bandas de 2.4 y $5 \mathrm{GHz}$, además del 802.11 ah que fue desarrollado para tratar escenarios de red de sensores inalámbricos (WSN), donde los dispositivos tienen restricciones de energía y requieren una comunicación de alcance relativamente largo, operando en banda de subgigahercios $(900 \mathrm{MHz})$. Ofrece velocidades de datos entre $1 \mathrm{Mbps}$ y $54 \mathrm{Mbps}$ para IEEE $802.11 \mathrm{a} / \mathrm{b} / \mathrm{g}$ que opera en un canal de $20 \mathrm{MHz}$. IEEE 802.11n, una enmienda a IEEE 802.11-2007, se basa en cuatro flujos de multi entrada y multi salida (MIMO) que admite velocidades de datos más altas en el rango de los 26 Mbps a 600 Mbps que operan en canales de 20 y $40 \mathrm{MHz}$. Para el inicio y establecimiento de la red, los Acces Point, difunden los paquetes que contienen el Identificador de conjunto de servicios (SSID) en la cabecera de los dispositivos conectados para que se pueda especificar la red.

WiMAX, proporciona diferentes clases de asignación de servicios y nivel de prioridades en su red $4 \mathrm{G}$ para garantizar que la QoS sirva para infraestructuras críticas, como la red inteligente. Sprint y su colaborador, Grid NetTM Inc., son dos de los principales defensores de la construcción de WiMAX para ofrecer soluciones de redes inteligentes [15].

La tecnología WSN ha sido ampliamente estudiada y ha ganado atención por parte del mundo académico y la industria en los últimos años [16]. Junto con su practicidad atractiva y su aplicabilidad diversa, la WSN diseñada para una amplia variedad de aplicaciones incluye servicios militares, seguridad y vigilancia, así como monitoreo y control en medio ambiente, industria, telemedicina/atención médica y automatización de viviendas/edificios. El despliegue de sensores tiende a crecer en cantidad por sistema, así como en tamaño y variedad en número de redes. El estudio realizado en [17] revela además que el bajo consumo de energía y la longevidad son indistintamente importantes dependiendo de dónde y para qué propósitos se aplican los WSN. Los estudios y literaturas sobre redes sociales y redes ad hoc han cubierto una variedad de temas de investigación. Pueden ser recursos instrumentales en la investigación de WLAN y WSN para la red inteligente. Las principales limitaciones del despliegue de una red de sensores inalámbricos incluyen consumo de energía [18], conectividad, sincronización [19], control de acceso al medio, enrutamiento, calidad de servicio, seguridad y privacidad.

La tecnología ZigBee se basa en el estándar de protocolo de comunicación IEEE 802.15.4 y se utiliza para redes de área personal o PAN [20, 21]. El estándar IEEE 802.15.4 tiene capas MAC y físicas de baja potencia. ZigBee fue desarrollado por la alianza ZigBee, y funciona para soluciones de comunicaciones fiables, de baja energía y económicas. El rango de comunicación del dispositivo ZigBee es relativamente pequeño (10-100 metros). Los detalles de la red y las capas de aplicación también están especificados por el estándar ZigBee. A diferencia de BLE, la capa de red proporciona enrutamiento multisalto. Hay tres tipos de dispositivos en una red de ZigBee: FFD (dispositivo completamente funcional), RFD (dispositivo funcional reducido) y un coordinador de ZigBee. Un nodo FFD también puede actuar como un enrutador. ZigBee admite topologías de estrella, árbol y malla. El esquema de enrutamiento depende de la topología. Otras características de ZigBee son el descubrimiento y mantenimiento de rutas, soporte para nodos que se unen / salen de la red, direcciones cortas de 16 bits y enrutamiento-multisalto. La alianza WiFi ha desarrollado recientemente "WiFi HaLow", que se basa en el estándar IEEE 802.11ah. Consume menos energía que un dispositivo WiFi tradicional y también tiene un alcance más largo. Esta es la razón por la cual este protocolo es adecuado para aplicaciones de Internet of Things. El alcance de WiFiHaLow es casi el doble que el de WiFi tradicional. Al igual que otros dispositivos WiFi, los dispositivos compatibles con WiFi HaLow también admiten la conectividad IP, que es importante para las aplicaciones IoT [22-23]. La Tabla 2 muestra un comparativo de las diferentes tecnologías de comunicación comentadas en el documento previamente.

\section{TECNOLOGÍAS SELECCIONADAS DE COMUNICACIÓN}

Como Internet of Things está creciendo muy rápidamente, hay una gran cantidad de dispositivos inteligentes heterogéneos que se conectan a Internet, estos dispositivos IoT funcionan con baterías, con recursos mínimos de computación y almacenamiento. Debido a su naturaleza limitada, hay varios desafíos de comunicación involucrados, que son los siguientes [24]: 
Tabla 2. Comparación de tecnologías IoT/M2M/WSN.

\begin{tabular}{|c|c|c|c|c|c|c|c|}
\hline $\begin{array}{c}\text { Technology } \\
\text { communication }\end{array}$ & Standard & Frequency Band & Network & \begin{tabular}{c|} 
Power \\
Consumption
\end{tabular} & Data Rate & Range & Access \\
\hline FIBER OPTIC & $\begin{array}{l}\text { SDH- MPLS } \\
\text { SONET }\end{array}$ & $\begin{array}{l}1.300-1.500 \\
\text { Nanometros. }\end{array}$ & WAN & $50 \mathrm{~mW}$ & $1.0-10.0 \mathrm{Gbps}$ & 100-1000 Km. & $\begin{array}{l}\text { TDMA/ } \\
\text { FDMA }\end{array}$ \\
\hline PLC-BLC & IEEE 802.3 Ethernet & \begin{tabular}{|l}
$125 \mathrm{kHz}$, \\
$13.56 \mathrm{MHz}$, \\
$902-928 \mathrm{MHz}$ \\
\end{tabular} & NAN & 10.0 Watts & $30 \mathrm{Mbps}$ & Up to $200 \mathrm{mtr}$. & OFDM \\
\hline BLE & IEEE 802.15.1 & \begin{tabular}{|l|}
$2.4 \mathrm{GHz}$ \\
Radio \\
\end{tabular} & HAN & $30 \mathrm{~mW}$ & $1 \mathrm{Mbps}$ & $15-30 \mathrm{mtr}$. & FHSS \\
\hline 6LoWPAN & IEEE 802.15.4 Radio & \begin{tabular}{|l}
$868 \mathrm{Mhz}$ (EU) \\
$915 \mathrm{Mhz}$ (USA) \\
2.4 Ghz (Glob) \\
\end{tabular} & HAN & $30 \mathrm{~mW}$ & 250 kbps & $10-100 \mathrm{mtr}$. & DSSS \\
\hline ZigBee & IEEE 802.15.4 Radio & $2.4 \mathrm{GHz}$ Radio & HAN & $30 \mathrm{~mW}$ & $250 \mathrm{kbps}$ & $30 \mathrm{~m}$ (indoors) & DSSS \\
\hline Wifi HaLow & IEEE 802.11 ah & 900MHz Radio & $\begin{array}{l}\text { HAN- } \\
\text { NAN }\end{array}$ & $100 \mathrm{~mW}$ & 1.0-600 Mbps & $0.2-1.0 \mathrm{~km}$ & RAW \\
\hline Cellular & $\begin{array}{l}\text { 3GPP, GSM, GPRS/ } \\
\text { UMTS (3G)LTE } \\
\text { (4G-5G) }\end{array}$ & $\begin{array}{l}\text { Commond } \\
\text { Cellular bands }\end{array}$ & WAN & $400 \mathrm{~mW}$ & $\begin{array}{l}250 \text { kbps- } \\
10 \text { Mbps }\end{array}$ & $0.2-3.0 \mathrm{~km}$ & DSSS \\
\hline SigFox & SigFox & \begin{tabular}{|l|}
$868 \mathrm{MHz}$ (EU) \\
$902 \mathrm{MHz}$ (USA) \\
\end{tabular} & HAN & $\begin{array}{l}10 \mathrm{~mW}- \\
100 \mathrm{~mW} \\
\end{array}$ & $\begin{array}{l}100 \text { bps (UL), } \\
600 \text { bps (DL) }\end{array}$ & $\begin{array}{l}10 \mathrm{~km} \text { (urban) } \\
50 \mathrm{~km} \text { (rural) }\end{array}$ & DSSS \\
\hline LoraWAN & LoraWAN & \begin{tabular}{|l|}
$169 \mathrm{MHz}$, \\
$433 \mathrm{MHz}$, \\
$868 \mathrm{MHz}$ (EU), \\
$915 \mathrm{MHz}$ (USA) \\
\end{tabular} & HAN & $\begin{array}{l}30 \mathrm{~mA} \text { Low } \\
\text { Power }\end{array}$ & 250 bps-50 kbps & $\begin{array}{l}2-5 \mathrm{~km} \text { (urban), } \\
15 \mathrm{~km} \text { (rural) }\end{array}$ & DSSS \\
\hline Weightless W-P & Weightless & $\begin{array}{l}\text { TV whitespace } \\
(400-800 \mathrm{MHz})\end{array}$ & HAN & $100 \mu \mathrm{W}$ & 1 kbps-10 kbps & 5 km (urban) & $\begin{array}{l}\text { TDMA/ } \\
\text { FDMA }\end{array}$ \\
\hline
\end{tabular}

1. Direccionamiento: Dado que millones de cosas inteligentes estarán conectadas a Internet, deberán identificarse a través de una dirección única, sobre la base de la cual se comunicarán entre sí. Para esto, necesitamos un espacio de direccionamiento y una dirección única para cada objeto inteligente.

2. Comunicación de baja potencia: La comunicación de datos entre dispositivos es una tarea que consume energía, especialmente la comunicación inalámbrica. Por lo tanto, necesitamos una solución que facilite la comunicación con bajo consumo de energía.

3. Protocolos: Enrutamiento con bajo requerimiento de memoria y patrones de comunicación eficientes.

Por lo general, los dispositivos de IoT se conectan a Internet a través de la dirección IP (Protocolo de Internet), que es compleja y requiere una gran cantidad de energía y memoria de los dispositivos de conexión. Los dispositivos IoT también se pueden conectar localmente a través de redes que no son IP, que consumen menos energía y se conectan a
Internet a través de una puerta de enlace inteligente. Los canales de comunicación no IP como Bluetooth, RFID y NFC son populares, pero tienen un alcance limitado (de un centímetro a dos metros). Por lo tanto, sus aplicaciones están limitadas a pequeñas redes de área personal. Las redes de área personal (PAN, por sus siglas en inglés) están siendo ampliamente utilizadas en aplicaciones de IoT, como los dispositivos portables conectados a teléfonos inteligentes. Para aumentar el alcance de tales redes locales, era necesario modificar la dirección IP para facilitar la comunicación de baja potencia utilizando la dirección IP. Una de las soluciones es 6LoWPAN, que incorpora IPv6 con redes de área personal de baja potencia. El rango de un PAN con 6LoWPAN es similar a las redes de área local, y el consumo de energía es mucho menor [1].

\section{CONCLUSIONES}

Se presentan, en este documento, las condiciones más críticas de la red que requiere comunicación para control y monitoreo de la generación eléctrica 
y de la distribución a través de una Infraestructura Avanzada de Medición (AMI).

La implementación de tecnologías Inalámbricas Redes de Sensores (WSN) para sistemas de potencia eléctrica, medición de suministro de energía, dependerán tanto de las distancias geográficas como de aplicación y velocidad de transmisión binaria. El tipo de red (WAN,NAN y HAN) que se quiera implementar y la consideración de los protocolos dedicados en cada escenario, exigirán diferentes niveles de QoS, latencia, cobertura y deberán ser considerados todos estos elementos.

A pesar de que PLC es una solución con bastantes ventajas debido a su despliegue, éste también tiene problemas de interferencia con el medio, como la atenuación, el ruido del medio y la distorsión que producen las conmutaciones de las cargas conectadas a la red de alimentación por la cual se quiere comunicar. Dado a que la línea eléctrica no se diseñó inicialmente para la transmisión de datos, es necesario abordar los problemas y desafíos de esta tecnología.

En redes que se conectan al servicio público con el sistema de distribución eléctrica con la última milla aplicando tecnología de Smart Metering, no requieren de un alto nivel de servicio, pero si requieren de confiabilidad y privacidad suficientes con el objetivo de monitorear de manera correcta el consumo de los clientes, para así emplear estrategias de respuesta a la demanda y mejorar el rendimiento del sistema.

Por lo tanto, la tecnología 4G actual y WiMAX IEEE 802.16, que admite el acceso inalámbrico de banda ancha fija y móvil es la más recomendable para proporcionar una mayor estabilidad y confiabilidad en la conectividad de la última milla IoT/ M2M.

Establecer una infraestructura de comunicaciones con un despliegue semejante a tecnologías celulares, cumpliendo con los requisitos de velocidad de datos y latencia, es lo que se debe planificar y considerar al desplegar sistemas tipo AMI para comunicación en ciudades Inteligentes.

Como trabajo futuro se pretende realizar un análisis que enfrente los costos de ancho de banda en Mbps versus las distancias en kilómetros que se desean cubrir a través de las redes extendidas tipo WAN.

\section{REFERENCIAS}

[1] F.K. Shaikh, S. Zeadally and E. Exposito. "Enabling Technologies for Green Internet of Things". IEEE Systems Journal. Vol. 11 No 2, pp. 983 - 994. 2017.

[2] ETSI Technical Committee Machine-toMachine. ETSI TS 102690 V1.1.1 (2011-10) Machine-to-Machine communications (M2M). Functional architecture. 2011.

[3] Telecommunication Standardization Sector of ITU. Applications of Wireless Sensor Networks in Next Generation Networks. Technical Paper. Series Y.2000. ITU-T. 2014.

[4] Telecommunication Standarization Sector of ITU. ITU-T telecommunication standardization sector of ITU. Recommendation ITU-T Y.2221. 2010.

[5] J. Zheng, D. Wenzhong Gao and L. Lin. "Smart Meters in Smart Grid: An Overview”. IEEE Green Technologies Conference. Denver, CO, USA. 2013.

[6] P. Kansal and A. Bose. "Bandwidth and Latency Requirements for Smart Transmission Grid Applications". IEEE Transactions on Smart Grid. Vol. 3 No 3, pp. 1344-1352. 2012.

[7] I. Decker Cassana, D. Dotta, M. Agostini and S.L. Zimath. "Performance of a Synchronized Phasor Measurements System in the Brazilian Power System". IEEE PES Power Systems Conference and Exposition. Atlanta, GA, USA. 2016.

[8] Fernando Ulloa, D. Carrizo and L. GarciaSantander. "Syncrhophasors Monitoring System in Electrical Network: Measurement during 8.4 Richter Seismic Activit". Conference proceedings IBIMA. Milan, Italia, pp. 125-129. ISBN: 978-0-99985510-2. 2018.

[9] C. Kalalas, L. Thrybom and J. AlonsoZarate. "Communications for Smart Grid Neighborhood Area Networks: A Survey". IEEE Access. Vol. 4, pp. 1469-1493. 2016.

[10] Informe Análisis Alternativas para la adopción de estándares para el Sistema de Medición, Monitoreo y Control. Norma Técnica de Calidad de Servicio para Sistemas de Distribución. Centro de Energía, Facultad de Ciencias Físicas y Matemáticas. Universidad de Chile. Diciembre de 2018. 
[11] W. Yuan, X. Wang and J.P. Linnartz. "A coexistence model of IEEE 802.15.4 and IEEE $802.11 \mathrm{~b} / \mathrm{g}$ ". 14th IEEE Symposium on Communications and Vehicular Technology in the Benelux. Delft, Netherlands. 2007.

[12] A. Mengi, S. Ponzelar and M. Koch. "The ITU-T G.9960 broadband PLC communication concept for smartgrid applications". IEEE International Conference on Smart Grid Communications (SmartGridComm). Dresden, Germany. 2017.

[13] F. Ulloa Vásquez y J.A. Delgado-Penin. "Acceso Fijo Radioeléctrico (FWA) Mediante HAPS. Evaluación del Comportamiento del Radio Enlace Digital en el Ámbito del IMT-2000”. Congreso y Revista “Telecom. I+D”. Madrid, España. 2002. ISBN: 92-9092-819-0.

[14] F. Ulloa-Vásquez, P. Olavarrieta Suárez and A. Soto Pereira. "Channel Predictions And Adaptive Code Modulation In Digital Broadband Communication Based On High Altitude Aeronautical Platform (Haap)". The Mediterranean Journal of Electronics and Communications. Vol. $8 \mathrm{~N}^{\circ}$ 2, pp. 360-365. 2015. DOI: 9.876/lpt2018-0112.

[15] Y. Feng, Y. Qian and R.Q. Hu. "Energy Efficient Self-Sustaining Wireless Neighborhood Area Network Design for Smart Grid". IEEE Transactions on Smart Grid. Vol. $6 \mathrm{~N}^{\mathrm{o}} 1$, pp. 220-229. 2015.

[16] J. Yick, B. Mukherjee and D. Ghosal. "Wireless sensor network survey". Computer Networks. Vol. $52 \mathrm{~N}^{\circ} 12$, pp. 2292-2330. 2008.
[17] K.R. Fowler. "The future of sensors and sensor networks survey results projecting the next 5 years". IEEE Sensors Applications Symposium. New Orleans, LA, USA. 2009.

[18] J. Blanckenstein, J. Klaue and H. Karl. "A Survey of Low-Power Transceivers and Their Applications". IEEE Circuits and Systems Magazine. Vol. $15 \mathrm{~N}^{\mathrm{o}}$ 3, pp. 6-17. 2015.

[19] Y.-P. Tian. "Time Synchronization in WSNs with Random Bounded Communication Delays". IEEE Transactions on Automatic Control. Vol. $62 \mathrm{~N}^{\mathrm{o}}$ 10, pp. 5445-5450. 2017.

[20] N.V. Rajeesh Kumar, C. Bhuvana and Y.S. Anushya. "Comparison of ZigBee and Bluetooth wireless technologies-survey". International Conference on Information Communication and Embedded Systems (ICICES). Chennai, India. 2017.

[21] P. Baronti, P. Pillai, V.W.C. Chook, S. Chessa, A. Gotta and Y.F. Hu. "Wireless sensor networks: a survey on the state of the art and the 802.15.4 and ZigBee standards". Computer Communications. Vol. $30 \mathrm{~N}^{\mathrm{o}} 7$, pp. 1655-1695. 2007.

[22] B. Shanmuga Sundaram. "A quantitative analysis of 802.11 a wireless standard". International Journal of Latest Research in Engineering and Technology. Vol. $2 \mathrm{~N}^{\mathrm{o}} 21$, pp. 26-29. 2016.

[23] W. Sun, M. Choi and S. Choi. "IEEE 802.11 ah: a long range 802.11 wlan at sub 1 ghz". Journal of ICT Standardization. Vol. $1 \mathrm{~N}^{\mathrm{o}} 1$, pp. 83-108. 2013.

[24] C.F. Hughes. "Bluetooth low energy". Ph.D. thesis. Arizona: Arizona State University. 2015. 